\title{
DETERMINING HEATING CAPACITY AND COEFFICIENT OF ENERGY TRANSFORMATION OF HYDRODYNAMIC SET
}

\author{
Breido Josif, Kalinin Alexey \& Kuchin Valery
}
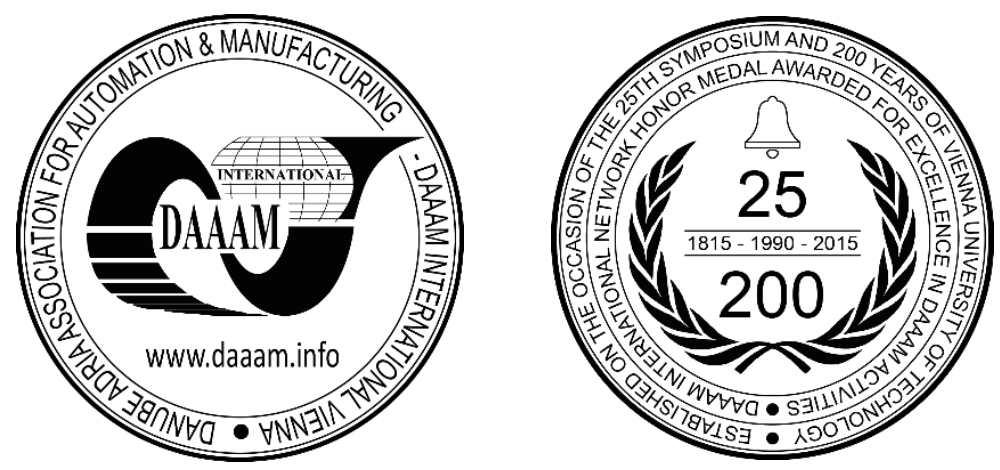

This Publication has to be referred as: Breido, J[osif]; Kalinin, A[lexey] \& Kuchin, V[alery] (2016). Determining Heating Capacity and Coefficient of Energy Transformation of Hydrodynamic Set, Proceedings of the 27th DAAAM International Symposium, pp.0600-0606, B. Katalinic (Ed.), Published by DAAAM International, ISBN 978-3-90273408-2, ISSN 1726-9679, Vienna, Austria

DOI: $10.2507 / 27$ th.daaam.proceedings.088

\begin{abstract}
In the work the developed constructive and technological diagram of the bench based on the frequency controlled electric drive of the pump set and SCADA system for experimental defining power characteristics of the hydrodynamic heaters (HDH) of liquid environments is described.

Based on methods of planning a multiple-factor experiment studies have been carried pit in various HDH operating modes. Preliminary theoretical calculations of the parameters of the HDH hydraulic operating mode have been carried out in the environment of the information-graphic system, at the set of factors similar to that of the pilot studies.

The mathematical dependences establishing interrelations of heating capacity and coefficient of energy transformation with the HDH constructive and operational parameters based on the pipe type reactor have been found.

The results of the studies and tests have been used when developing specifications for designing and producing the HDH prototypes.
\end{abstract}

Keywords: hydrodynamic heater, bench, model, studies, tests, methods of planning a multifactor experiment

\section{Introduction}

One of the trends of increasing the efficiency of power systems is obtaining thermal energy from internal energy of liquid environments by means of activation with external sources (for example, an electric drive). This trend is connected with designing, studying and producing hydrodynamic heaters (HDH). Their internal energy is taken from the liquid environment (the so-called "working liquid") in the course of activation by means of electromechanical impact.

In 1993 in the USSR and the USA the inventions of Yu.S. Potapov [1] and James Griggs [2] were patented which could change the technological role of water in power technologies. Water can be used not only as an effective energy carrier but also as an available and cheap energy resource.

The analysis of these experimental tests and technical characteristics of HDH permits to find a set of unresolved problems which slow down a wide use of vortex power effects in power technologies and sets. These problems are caused by the absence of high-quality and reliable bench studies and certification tests of the finished samples of hydrodynamic converters of energy and theoretical generalizations accompanying them. There is no design methodology without which designing and producing a number of standard sizes HDH is impossible [3-6]. At present the processes of transforming 
electric energy into mechanical energy with the subsequent transforming mechanical one into thermal one are not studied completely.

Today the development of methods of HDH designing and determining its optimum parameters providing the maximum heating capacity and coefficient of transformation of energy is topical.

\section{Test bench for studying HDH power characteristics}

The authors have developed a HDH constructive and technological diagram. The test bench for studying the HDH power characteristics has been developed [7]. The bench contains an automated system of scientific studies for detecting the regularity of transforming electric energy into mechanical one with the subsequent transforming it to thermal energy [8]. The task has been set: on the basis of multifactor experiment to find the HDH optimum parameters providing the maximum heating capacity and coefficient of energy transformation. Then to obtain mathematical dependences establishing interrelations of heating capacity and coefficient of energy transformation with the HDH constructive and operational parameters.

The HDH constructional units were to provide the working liquid circulation in the closed heat-generating contour. At this in the pipe reactor (Figure 1, [9]) with the liquid vortex motion the effect of cavitation is developed. As a result the allocation of internal energy from the working liquid in the form of heat takes place.

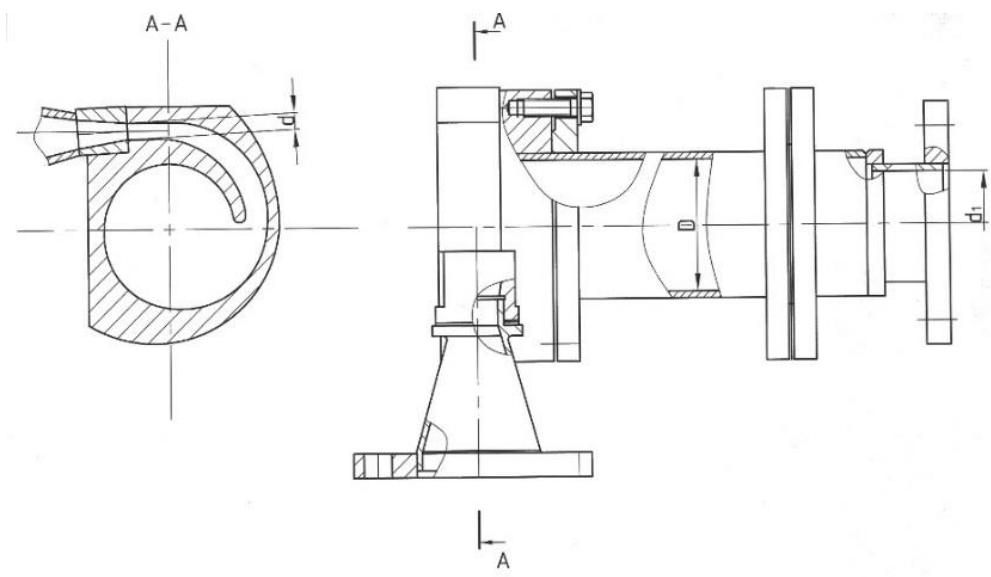

Fig. 1. Hydrodynamic heater sketch

The technological diagram of the test bench of the HDH system with a pipe reactor for studying power characteristics of the experimental series of the hydrodynamic heater is given in Figure 2. The bench permitted to carry out a complex of tasks of planning, collecting and processing measurements of the electric, hydrodynamic and heat-physical processes which were taking place in the HDH elements. In the diagram (Figure 2) there are two contours of the working liquid circulation: a heat-generating contour and a load contour. In the heat-generating contour where from the working liquid the internal energy releases, there is a circulation pump (pos. 1), a hydrodynamic heater (pos. 2) and a heat accumulator (pos. 3) representing a tank store. The load contour serves for accumulation or consumption of the released thermal energy. It contains a circulation pump (pos. 1), a heat accumulator (pos. 3) and an air-heating unit which part is a heater (pos. 4) and a fan (pos. 5). The shutoff valves were used for selecting a joint or a separate operating mode of the contours. At the set points of the technological diagram sensors for digital measurements of the working liquid parameters in both contours, as well as operating modes of the electric motor of the circulation pump are mounted.

The circulation pump unit (pos. 1) with the frequency controlled drive provides the working liquid movement both through the HDH and through the load contour. Rated capacity of the electric motor of the pump unit is spent for the vortex motion of the working liquid combined with the continuous cavitation and overcoming hydrodynamic resistance of all the units of the heat-generating and load contours.

The frequency controlled electric drive of the pump is needed for supporting in the course of the experiment of the working liquid pressure at the intake branch pipe of the $\mathrm{HDH}$ at the constant level. The working liquid pressure determines the value of the water stream specific energy therefore its values are to be selected from the pump characteristic "Pressure head - Flow rate" at the maximum frequency of rotation in the vicinity of the working zone where the efficiency and power of the pump have the maximum value. At this the water extreme mechanical activation in the HDH with emission of excess heat is provided. For controlling the course of the experiment it is expedient to use a system of dispatching control and data collection. It is not only to accumulate the measured values, to watch the experiment course in the parameters set by the experimenter, but also to set and support the frequency of circulation pump engine shaft rotation.

The HDH bench is equipped with measuring inserts for digital measuring the working liquid temperature, pressure and consumption. Measuring inserts for measuring the flow rate (pos. $1 \mathrm{~g}$ and $2 \mathrm{~g}$ ) represent a straight section of the pipeline without local hydraulic resistance on which the flow rate sensor is mounted. Measuring inserts for measuring pressure and temperature of the working liquid represent selected devices for mounting sensors of pressure (pos. 1p, 2p, 3p) and temperature sensors (pos. 1t, 2t, 3t, 4t, 5t) in the set sections of the heat-generating and load contours. 


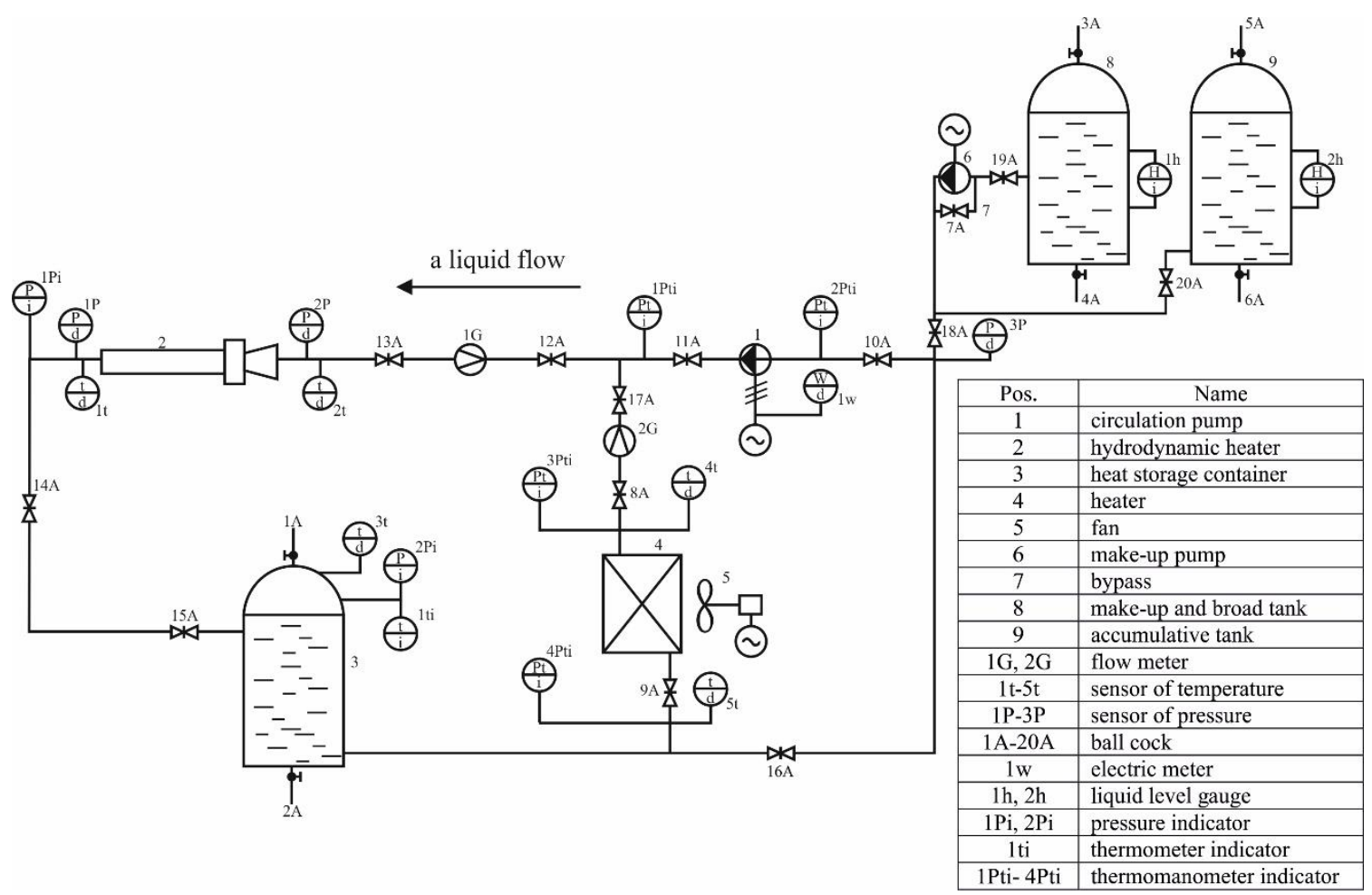

Fig. 2. Technological network of the bench

The system of controlling and collecting experimental data has been selected the SCADA-system Genesis32 permitting to measure and accumulate in the database in discrete intervals of real time variable conditions of the turbulent movement of the working liquid.

\section{Methodology of studying}

The task of determining heating capacity and coefficient of energy transformation and their maxima as a function of the HDH power and geometrical parameters has been set.

The criterion function for studying the mechanism of the HDH action is as follows:

$$
\mathrm{Q}=\mathrm{F}(\mathrm{X} ; \mathrm{P}) \text {, at } \mathrm{P}=\text { const, }
$$

where $\mathrm{X}=\left(\mathrm{X}_{1}, \mathrm{X}_{2}, \ldots, \mathrm{X}_{\mathrm{k}}\right)$ is the vector of variable factors;

$\mathrm{P}=\left(\mathrm{P}_{1}, \mathrm{P}_{2}, \mathrm{P}_{3}, \ldots, \mathrm{P}_{\mathrm{m}}\right)$ is the vector of fixed parameters.

The pipe type reactor HDH constructive and mode parameters have been selected as variable factors:

1) $X_{1}$ - the pipe reactor length, $\mathrm{mm}$;

2) $X_{2}$ - internal diameter of the pipe reactor, mm;

3) $X_{3}$ - the working liquid pressure at the spiral supply intake, MPa;

4) $X_{4}$ - the time of the circulation pump operation, $h$.

The HDH qualitative and quantitative parameters have been defined as fixed factors:

1) $P_{1}-$ the brake unit type;

2) $P_{2}$ - the direction of the whirlwind (right rotary, left rotary);

3) $\mathrm{P}_{3}$ - physical-and-chemical properties of the working liquid;

4) $\mathrm{P}_{4}$ - the circulation pump type;

5) $\mathrm{P}_{5}$ - the circulation electric engine power, $\mathrm{kW}$;

6) $\mathrm{P}_{6}-$ the working liquid starting temperature, ${ }^{\circ} \mathrm{C}$;

7) $\mathrm{P}_{7}$ - the working liquid mass in the volume of the storage vessel, kg;

8) $\mathrm{P}_{8}$ - the spiral supply intake area diameter, $\mathrm{mm}$;

9) $\mathrm{P}_{9}$ - the cavitator exit area diameter, $\mathrm{mm}$.

For experimental obtaining a mathematical model connecting the HDH criterion function of GDN, the variable factors and the state parameters the plan of experiments based on the Latin squares and the multifactor equations of Protodyakonov-Teder was used [10].

The methodological advantage of such an approach consists in the absence of initial connection between the experiment plan and the form of a possible mathematical model. It gives an opportunity to the experimenter to consider more completely the features of the studied phenomenon on the basis of the creative judgment of both the objective and 
the obtained results of the experiments. At this the correctness of selecting the models of private functions and the generalizing dependence is estimated by statistical criteria [11].

The pragmatic advantage of such an approach consists, first of all, in the sharp reduction of the needed number of experiments. Another advantage of this methodology is the possibility of expanding the limits of applicability of the generalizing mathematical model independent on physical limits of changing the studied factors [12].

Such a plan of studies which resolution is provided at the minimum number of experiments has been chosen. In the four-factorial studying of the phenomena and the processes such an opportunity belongs to the plan at three levels in which it is necessary to carry out only 9 experiments, every time with a new combination of the factors values. The type of such a plan with code designations of the factors values is given in Table 1.

\begin{tabular}{|c|c|c|c|c|c|}
\hline \multirow{2}{*}{ Experiment No. } & \multicolumn{4}{|c|}{ Variable factors } & \multirow{2}{*}{$\frac{\text { Criterion function }}{\mathrm{Q}}$} \\
\hline & $\mathrm{X} 1$ & $\mathrm{X} 2$ & X3 & $\mathrm{X} 4$ & \\
\hline 1 & 1 & 1 & 3 & 1 & Q1 \\
\hline 2 & 2 & 2 & 2 & 1 & Q2 \\
\hline 3 & 3 & 3 & 1 & 1 & Q3 \\
\hline 4 & 1 & 2 & 1 & 2 & Q4 \\
\hline 5 & 2 & 3 & 3 & 2 & Q5 \\
\hline 6 & 3 & 1 & 2 & 2 & Q6 \\
\hline 7 & 1 & 3 & 2 & 3 & Q7 \\
\hline 8 & 2 & 1 & 1 & 3 & Q8 \\
\hline 9 & 3 & 2 & 3 & 3 & Q9 \\
\hline
\end{tabular}

Table 1. Plan of four-factorial experiment at three levels in the abstract space of variables (Latin square)

Each line of this plan represents specific conditions of the experiment. In the same line the result is registered. From the results the samples for three-point private dependences of heating capacity on each of the factors are formed: $\mathrm{q}_{1}\left(\mathrm{X}_{1}\right)$, $\mathrm{q}_{2}\left(\mathrm{X}_{2}\right), \mathrm{q}_{3}\left(\mathrm{X}_{3}\right), \mathrm{q}_{4}\left(\mathrm{X}_{4}\right)$. As to the zero value of private dependences for physical reasons there can correspond a zero value of the generalizing multifactor dependence, it should be formed as the product of private dependences $\mathrm{q}_{\mathrm{i}}(\mathrm{Xi})$ in the form:

$$
Q_{g}=\frac{\prod_{i=1}^{k} q_{i}\left(X_{i}\right)}{q_{a v}^{k-1}}
$$

where $Q_{g}$ is a multifactor function of Protodyakonov;

$\mathrm{q}_{\mathrm{i}}(\mathrm{Xi})$ is a private function of one argument for each of the studied factors;

$\mathrm{k}$ is the number of private functions (factors); executed.

$\mathrm{q}_{\mathrm{cp}}$ is the average value of all the considered results of the experiment where the private functions normalizing is

For the indirect measuring of the HDH heat capacity in the i-th experiment the following expression has been used:

$$
Q=k \cdot G \cdot c_{p}\left(\frac{d t}{d \tau}\right)_{i}
$$

where $\mathrm{Q}$ is $\mathrm{HDH}$ heat capacity, $\mathrm{kW}$;

$\mathrm{k}=1.163$ is the coefficient the thermal stream dimension transformation from $\mathrm{kcal} / \mathrm{h}$ into $\mathrm{W}$;

$\mathrm{G}$ is the working liquid mass in the volume of the circulation contour, $\mathrm{kg}$;

$\mathrm{c}_{\mathrm{p}}$ is the working liquid specific heat capacity, $\mathrm{kcal} / \mathrm{kg}^{\circ} \mathrm{C}$;

$\left(\frac{d t}{d \tau}\right)_{i}$ is the working liquid heating temp in the $\mathrm{HDH}$ reactor in the $\mathrm{i}$-th experiment.

To express the HDH heat capacity in the form of the criterion P-function $Q(L, d, P, t)$ the following four private functions of one argument heat capacity have been selected:

$q_{1}(L)$ is the dependence of heat capacity on the pipe reactor length;

$q_{2}(d)$ is the dependence of heat capacity on the reactor internal diameter;

$q_{3}(P)$ is the dependence of heat capacity on the pressure at the spiral supply intake;

$q_{4}(t)$ is the dependence of heat capacity on the pump operation time.

To check the adequacy of the dependence the coefficient of nonlinear multiple correlation has been used [13]:

$$
R=\sqrt{1-\frac{(n-1) \sum_{i=1}^{n}\left(q_{e i}-q_{t i}\right)^{2}}{(n-k-1) \sum_{i=1}^{n}\left(q_{e i}-q_{a v}\right)^{2}}}
$$

where $\mathrm{n}$ is the number of described points 9 for the accepted experiment plan $n=3$ ); 
$\mathrm{k}$ is the number of acting factors: for private approximating functions $\mathrm{k}=1$, and for generalizing function $\mathrm{k}=4$ ); $q_{\ni i}$ is the experimental value of the result, i.e. heat capacity;

$q_{t i}$ is the theoretical (calculated) value of the result;

$q_{c p}$ is the average experimental value.

Theoretical calculations have been carried out in the environment of the information and graphic GID-99w system on the same set of factors as in the HDH pilot studies. The GID-99w has been developed at Karaganda State Technical University and intended for imitating modeling and the spatial technological analysis of the operational and emergency hydraulic operation of the heat-supplying systems of a variable technological structure and an arbitrary set of the standard heat-mechanical equipment [14-16].

The importance of the coefficient of correlation and of the checked approximating function for the $95 \%$ level of reliability is defined by the inequality [13]:

$$
t_{r}=\frac{R \sqrt{n-k-1}}{1-R^{2}}>2 .
$$

\section{Experimental results processing}

The adequacy of private functions of heating capacity was carried out by means of coefficient $\mathrm{R}$ of nonlinear multiple correlations by the criterion of importance $t_{R}(5)$ with the $95 \%$ level of reliability. The results of the adequacy estimates are given in Table 2.

\begin{tabular}{|c|c|c|c|c|}
\hline $\mathbf{q}_{\mathbf{i}}$ & $\mathbf{R}$ & Importance by $\mathbf{R}$ & $\mathbf{t}_{\mathbf{R}}\left(\mathbf{t}_{\mathbf{R}}>\mathbf{2}\right)$ & Importance by $\mathbf{t}_{\mathbf{R}}>\mathbf{2}$ \\
\hline $\mathrm{q}_{1}(\mathrm{~L})$ & 0.7299 & Important & 1.5622 & Unimportant \\
\hline $\mathrm{q}_{2}(\mathrm{~d})$ & 1 & Important & $\infty$ & Important \\
\hline $\mathrm{q}_{3}(\mathrm{P})$ & 0.9999 & Important & 4018579.401 & Important \\
\hline $\mathrm{q}_{4}(\mathrm{t})$ & 0.9999 & Important & 210614020.5 & Important \\
\hline
\end{tabular}

Table 2. Heat capacity $\mathrm{q}_{\mathrm{i}}$ of private functions estimates

The resultant approximating function of the pipe type reactor HDH heating capacity formed by the structure of Protodyakonov multifactor function has obtained the following expression:

$$
\begin{aligned}
& Q(L, d, P, t)=\left(5 \cdot 10^{-6} L^{2}-0.0114 L+12.035\right) \cdot\left(-0.0002 d^{2}+0.0304 d+4.9627\right) \\
& \left(-67.94 P^{2}+87.376 P-21.631\right) \cdot\left(-2.7269 t^{2}+6.8838 t+2.1474\right) / 5.883^{3}
\end{aligned}
$$

At this the function appeared important $\left(R=0.9974: t_{R}=197.9978>>2\right)$ that permitted to use it in the physically expedient range of parameters $(\mathrm{L}, \mathrm{d}, \mathrm{P}, \mathrm{t})$, that is $714 \mathrm{~mm} \leq \mathrm{L} \leq 1500 \mathrm{~mm} ; 51 \mathrm{~mm} \leq \mathrm{d} \leq 150 \mathrm{~mm} ; 0.6 \mathrm{Mpa} \leq \mathrm{P} \leq 0.8 \mathrm{MPa}$; $1 \mathrm{~h} \leq \mathrm{t} \leq 2 \mathrm{~h}$.

The experimental and calculated values of the HDH heating capacity obtained by (7) and used for calculation of the criterion of importance are given in Table 3. Numerical studies for the function extremum (7) by the method of complete enumeration of the parameters $(\mathrm{L}, \mathrm{d}, \mathrm{P}, \mathrm{t})$ space with a small step of change has permitted to establish the optimum values of the variable factors and extreme value of the HDH heating capacity given in Table 4.

The obtained extreme value of heating capacity of the experimental series has made $8.118 \mathrm{~kW}$ or $6980.22 \mathrm{kcal} / \mathrm{h}$. At this thermal losses through the thermal isolation of the circulating contour have made $204.319 \mathrm{kcal} / \mathrm{h}$. Then the HDH complete heating capacity taking into account thermal losses through the isolation of the circulating contour has made $7184.542 \mathrm{kcal} / \mathrm{h}$.

By the similar technique the resultant approximating function of coefficient of energy transformation has been obtained. The coefficient of energy transformation represents the HDH power efficiency and is defined from the expression:

$$
K n_{e i}=Q_{i} / P_{i} \cdot \eta_{i}
$$

where $\mathrm{Q}_{i}, \mathrm{P}_{\mathrm{i}}, \eta_{\mathrm{i}}$ are respectively the HDH heat capacity, power consumed by the pump aggregate and the pump aggregate efficiency in the mode of the i-th parallel experiment on the current line of the matrix of planning; the efficiency established on the physical prototype of the HDH system of the pump aggregate with $7.5 \mathrm{~kW}$ power in the nominal mode (at the maximum efficiency) is equal $\eta=0.63$.

$$
\begin{aligned}
& K n_{m}(L, d, P, t)=\left(-2 \cdot 10^{-7} L^{2}+0.0004 L+1.0773\right) \cdot\left(3 \cdot 10^{-6} d^{2}-0.0033 d+1.5368\right) \\
& \left(-5.6123 P^{2}+6.9644 P-0.8747\right) \cdot\left(-0.3069 t^{2}+0.8619 t+0.6949\right) / 1.246144^{3}
\end{aligned}
$$




\begin{tabular}{|c|c|c|c|c|c|c|c|}
\hline Experiment No. & $\mathbf{L}, \mathbf{~ m m}$ & $\mathbf{d}, \mathbf{m m}$ & $\mathbf{P}, \mathbf{M P a}$ & $\mathbf{t}, \mathbf{h}$ & $\mathbf{Q e}, \mathbf{k W}$ & $\mathbf{Q t}, \mathbf{k W}$ & Relative error, \% \\
\hline 1 & 714 & 51 & 0.70 & 1 & 7.3101 & 7.4634 & 2.0541 \\
\hline 2 & 1200 & 100 & 0.60 & 1 & 6.5444 & 6.5416 & 0.0434 \\
\hline 3 & 1500 & 150 & 0.50 & 1 & 5.0583 & 4.8784 & 3.6867 \\
\hline 4 & 714 & 100 & 0.50 & 1.5 & 6.3165 & 6.1068 & 3.4334 \\
\hline 5 & 1200 & 150 & 0.70 & 1.5 & 5.7280 & 5.4203 & 5.6773 \\
\hline 6 & 1500 & 51 & 0.60 & 1.5 & 6.9680 & 7.3099 & 4.6780 \\
\hline 7 & 714 & 150 & 0.60 & 2 & 5.4955 & 5.0436 & 8.9585 \\
\hline 8 & 1200 & 51 & 0.50 & 2 & 3.8404 & 4.1524 & 7.5139 \\
\hline 9 & 1500 & 100 & 0.70 & 2 & 5.6861 & 5.6987 & 0.2224 \\
\hline \multicolumn{7}{|l|}{} \\
\hline
\end{tabular}

Table 3. Experimental Qe and theoretical Qt values of the HDH heat capacity predicted by the Q(L,d,P,t) function

\begin{tabular}{|c|c|c|c|c|}
\hline $\mathbf{Q}, \mathbf{k W}$ & $\mathbf{L}, \mathbf{m m}$ & $\mathbf{d}, \mathbf{m m}$ & $\mathbf{P}, \mathbf{M P a}$ & $\mathbf{t}, \mathbf{h}$ \\
\hline 8.118 & 714 & 76 & 0.65 & 1.25 \\
\hline
\end{tabular}

Table 4. Extreme values of heat capacity parameters

In Table 5 there are presented experimental and calculated values of the coefficient of energy transformation obtained by (8) and used for calculating the criterion of importance.

\begin{tabular}{|c|c|c|c|c|c|c|c|}
\hline Experiment No. & $\mathbf{L}, \mathbf{m m}$ & d, $\mathbf{m m}$ & $\mathbf{P}, \mathbf{M P a}$ & $\mathbf{t}, \mathbf{h}$ & Кпе & KIIt & Relative error, \% \\
\hline 1 & 714 & 51 & 0.70 & 1 & 1.3906 & 1.4016 & 0.7844 \\
\hline 2 & 1200 & 100 & 0.60 & 1 & 1.2985 & 1.3015 & 0.2281 \\
\hline 3 & 1500 & 150 & 0.50 & 1 & 1.0608 & 1.0591 & 0.1537 \\
\hline 4 & 714 & 100 & 0.50 & 1.5 & 1.2558 & 1.2592 & 0.2674 \\
\hline 5 & 1200 & 150 & 0.70 & 1.5 & 1.1907 & 1.1802 & 0.8872 \\
\hline 6 & 1500 & 51 & 0.60 & 1.5 & 1.4454 & 1.4534 & 0.5489 \\
\hline 7 & 714 & 150 & 0.60 & 2 & 1.1068 & 1.1051 & 0.1560 \\
\hline 8 & 1200 & 51 & 0.50 & 2 & 1.2968 & 1.2951 & 0.1351 \\
\hline 9 & 1500 & 100 & 0.70 & 2 & 1.1699 & 1.1682 & 0.1489 \\
\hline \multicolumn{5}{|l|}{ Average value, $x_{\mathrm{cp}}$} & 1.246 & 1.247 & \\
\hline \multicolumn{5}{|c|}{ Maximum value, $x_{\max }$} & 1.445 & 1.453 & \\
\hline \multicolumn{5}{|c|}{ Minimum value, $x_{\min }$} & 1.061 & 1.059 & \\
\hline \multicolumn{5}{|c|}{ Variation range, $\mathrm{R}=\mathrm{x}_{\max }-\mathrm{X}_{\min }$} & 0.385 & 0.394 & \\
\hline \multicolumn{5}{|c|}{ Mean linear deviation, $a=\frac{1}{n} \sum_{i=1}^{n}\left|x_{i}-\bar{x}\right|$} & 0.1014 & 0.1057 & \\
\hline \multicolumn{5}{|c|}{ Mean-square deviation, $\sigma=\sqrt{\frac{1}{n} \sum_{i=1}^{n}\left(x_{i}-\bar{x}\right)^{2}}$} & 0.1134 & 0.1175 & \\
\hline \multicolumn{5}{|c|}{ Coefficient of correlation } & \multicolumn{2}{|c|}{0.9994} & \\
\hline
\end{tabular}

Table 5. Experimental $\kappa_{\Pi_{e}}$ and theoretical $\kappa_{n_{t}}$ values of the coefficient of energy transformation predicted by the $\mathrm{K}_{\Pi_{\mathrm{t}}}(\mathrm{L}, \mathrm{d}, \mathrm{P}, \mathrm{t})$ function

The HDH parameters extreme values for the function of the coefficient of transformation are presented in Table 6 .

\begin{tabular}{|c|c|c|c|c|}
\hline KII & L, $\mathbf{~ m m}$ & d, $\mathbf{~ m m}$ & $\mathbf{P}, \mathbf{M P a}$ & $\mathbf{t}, \mathbf{h}$ \\
\hline 1.5159 & 1000 & 51 & 0.6 & 1.4 \\
\hline
\end{tabular}

Table 6. Extreme values of the coefficient of energy transformation parameters 


\section{Conclusion}

As a result of the carried out studies the following results have been obtained:

- the HDH constructive and technological diagram and the experimental bench have been developed for studying the HDH power characteristics of liquid environments with an automated system of the HDH scientific studies;

- the system of automatic control of the bench electric equipment with the frequency-controlled electric drive for detecting the regularity of transforming electric energy into mechanical one with the subsequent transforming it to thermal energy has been developed;

- there have been carried out based on the methods of planning a multifactor experiment studies in the HDH various operating modes [17-20];

- the processes of transforming electric energy into mechanical one and mechanical energy into thermal one with finding the extreme value of heating capacity of the experimental series have been investigated;

- the methods of establishing the HDH optimum parameters providing the maximum heating capacity and coefficient of energy transformation on the basis of multifactor experiment have been defined;

- the mathematical dependences establishing interrelations of heating capacity and coefficient of energy transformation with the constructive and operational parameters of the HDH with the pipe type reactor have been found.

\section{References}

[1] RF patent No. 2045715, MPC F25B29/00. The heat generator for liquid heating [Text] /the patent holder Potapov Yu.S. (RU). No. 93021742/06; appl. 26.04.1993; publ. 10.10.1995.

[2] U.S. Pat. No. 5188090 A. Apparatus for heating fluids / James L. Griggs - application 08.04.1991, № US 07/682,003; published by 23.02.1993.

[3] Merkulov, A. (1997).Vortex effect and its application in equipment. Samara: Optima, 348 p.

[4] Yong Li \& Mian Lin (2010). Hydrodynamic coefficients induced by waves and currents for submerged circular cylinder, Procedia Engineering, Volume 4, 2010, pp. 253-261.

[5] Santhosh Kumar Gugulothu, P.R. Vijaya Kumar, P. Deekshith (2012). Exploring Cavitating Phenomenon With and Without Ultrasonic Transducer, Procedia Engineering, Volume 38, 2012, pp. 154-164

[6] Furmakov, E. (2004). Can hydrodynamic heat generators work superefficiently? Coll. "Problems of studying the Universe": St-Pb. 21 p.

[7] Breido, I., Kalinin, A., \& Tomilova, N. Field and simulation study of the hydrodynamic heater for fluid environments, IEEE Xplore, in press.

[8] Kalinin, A. (2014). Automated system of scientific studying hydrodynamic heaters. Proc. of the international scientific and methodical conference Informatization of Engineering Education, ISBN 978-5-7046-1535-4, April 10-11, 2014 NIU MEI, Moscow - M.: MEI publishing house, pp. 359-362.

[9] RK patent No. 6900 PK KZ B. Hydrodynamic heater [Text] / Karasyov N.I., Kuchin V.N., Okrut I.I. Bull.No. 10, 2002.

[10] Protodyakonov, M. \& Teder, R.(1970). Methodology of rational planning experiment M.: Nauka, 1970.

[11] Zedginidze, I. (1976). Planning experiment for studying multicomponent systems. M.: Nauka, 390 p.

[12] Brodsky, V. (1976). Introduction to factorial planning of experiment - M.: Nauka, 224 p.

[13] Rumshinsky, L. (1971). Mathematical processing of experimental results - The main editorial office of physicalmathematical science of publ.house "Nauka", $192 \mathrm{p}$.

[14] Karasyov, N. (2002). Solver of problems of imitating modeling the modes of large heat supplying systems//Power, telecommunications and higher education in modern conditions: 3rd International scientific and practical conference, ISBN 9965-9206-0-5, Almaty: APEC, pp. 24-28.

[15] Karasyov, N. \& Kalinin, A. (2010). Modeling stationaryh flow distribution in the IGS GID-99w environemtn. Proc. of int.symp. «Information-and-communication technologies in industry, education and science», ISBN 9965-04599-2, October 28-29, 2010 in 2 parts. Part 1. RK Ministry of Education and Science, KSTU, pp.163-165.

[16] Tomilova, N., Kalinin, A., \& Tsok, A. (2012). Mathematical models of stationary hydraulic modes of heat supplying systems in terms of regime situations//University Proc., Iss. 1 / KSTU. Karaganda, pp. 92-95, ISSN 1609-1825

[17] RK patent No. 30705. MPC F24H 1/10, F24J 3/00, F24H 7/00, F24D 3/02. Mechanoactivated heat generator [Text] / Kuchin B.H. (KZ); Bekturganov N. S. (KZ); Isaev V. L. (KZ); Kalinin A.A. (KZ); Yurchenko V. V. (KZ) - No. 2014/1278.1; appl. 03.10.2014; publ. 15.12.2015, Bull. No. 12(I), 2015.

[18] Kuchin, V., Yurchenko, V., \& Kalinin, A. (2015). Pilot studies of power characteristics of trial installations of environmentally friendly hydrodynamic heaters of liquid environments. Monograph, ISBN 978-601-296-932-0, Karaganda: Publishing house of KSTU, 78 p.

[19] Kuchin, V., Yurchenko, V., Kalinin A., \& Mergenov, A. (2015). Pilot studies of power characteristics of hydrodynamic heaters of liquid environments. Bull. of the Kazakhstan National Academy of Natural Sciences No. $1 / 2015$, pp. 32-34, ISSN 2222-3851.

[20] Kuchin, V., Yurchenko, V., Kalinin, A., \& Bekturganov, N. (2015). Studying cavitation process of hydrofynamic heater. Bull. of Kazakhstan National Academy of Natural Science. No. 2/2015, pp. 55-58, ISSN 2222-3851. 